\title{
SOX and light sterile neutrinos
}

\section{Gioacchino Ranucci ${ }^{1}$}

Istituto Nazionale di Fisica Nucleare

Via Celoria 16, 20133 Milano, Italy

E-mail: gioacchino.ranucci@mi.infn.it

The large size and the very low radioactive background achieved by the solar neutrino detector Borexino at the Gran Sasso Laboratory represent a unique opportunity to probe the existence of neutrino oscillations into new sterile components exploiting properly realized antineutrino and neutrino artificial sources. In this work, I briefly sketch the distinctive features of the SOX experiment, a program for the search of sterile neutrinos with Borexino that will be realized by deploying close to the detector an intense ${ }^{144} \mathrm{Ce}^{-144} \mathrm{Pr}$ anti-neutrino source and, possibly in a second stage, a ${ }^{51} \mathrm{Cr}$ neutrino source.

Neutrino Oscillation Workshop

4 - 11 September, 2016

Otranto (Lecce, Italy)

\footnotetext{
${ }^{1}$ Speaker on behalf of SOX collaboration
} 


\section{Introduction}

The successful saga of neutrino oscillation, culminated in the 2015 Nobel Prize, paves the way to a future, rich and diverse experimental program of precision experiments tasked to complete the determination of the elements of the PNMS oscillation matrix with unprecedented accuracy, and to unravel the yet undiscovered features of neutrino properties. Therefore, mass hierarchy determination, octant of $\theta_{23}$, violating $\delta_{\mathrm{cp}}$ phase and improved precision of the massmixing parameters (mixing angles as well as squared mass differences), are the core of the ambitious worldwide neutrino oscillation program shaped for the forthcoming two decades. At the same time the Dirac or Majorana nature of the neutrino mass term, together with its absolute value, will be probed by a suite of dedicated experiments.

The elegant and successful scheme of the three flavor oscillation paradigm, however, does not exclude deviations from the SSM, taking also into account that per se the non zero mass implied by the oscillation mechanism is already a (modest) detour from the SSM itself, in turn pointing to another source of mass at a new high energy scale, indirectly responsible for the tiny neutrino masses (possibly of Majorana nature), for example a la seesaw mechanism.

Moreover, scenarios beyond the standard model contemplate the existence of the so called non standard neutrino interactions (NSI), and, of immediate relevance for the present discussion, of sterile right handed neutrinos, which may occur at vastly different scales.

In particular, there is no a priori theoretical motivation to find a hypothetical sterile state at the $\mathrm{eV}$ scale (this is the scale referred when talking of light sterile neutrinos). Rather, it is the occurrence of few long standing anomalies at small L/E which may be interpreted as mixing of one or more sterile neutrinos at the $\mathrm{eV}$ scale with the known active states.

In a short schematic list, these anomalies are: a) the long standing LSND puzzle [1] (anomalous excess of anti- $\nu_{\mathrm{e}}$ in an anti- $v_{\mu}$ beam), b) the cumulative observations of less than expected events in the reactor experiments with baseline from few $\mathrm{m}$ to $1 \mathrm{~km}$ ("reactor anomaly") [2], and c) the similar evidence of count rate deficit in the ${ }^{51} \mathrm{Cr}$ and ${ }^{37} \mathrm{Ar}$ source tests in the Gallium solar $v$ detectors ("Gallium anomaly") [3].

The suggestive coincidence that these anomalies point to $\sim 1 \mathrm{eV}$ mass scale has boosted the interest on light sterile neutrinos, which is thus a well experimentally motivated area of investigation.

In this context, the SOX project aims to play a central role in clarifying the puzzling experimental situation through a straightforward test based on a high intensity radioactive source deployed close to the Borexino detector.

\section{Shedding light on the sterile hypothesis with a source based test}

A source experiment conceived to test the light sterile neutrinos scenario is characterized by two main requirements: the exploitation of a pure source of neutrinos or antineutrino in the range $0.1-10 \mathrm{MeV}$ and the capability to measure the interaction rate as a function of the distance from the source itself. Actually, both requirements could be fulfilled using as neutrino (or 
antineutrino) origin either a reactor core (antineutrinos only) or an on purpose radioactive source (which could be chosen to emit neutrinos or antineutrinos).

In both cases the fundamental capability to reconstruct the interaction rate as function of the distance can be accomplished according two possible options: a movable detector from a few up to $\sim 20 \mathrm{~m}$ from the source, or the exploitation of a large detector, either segmented or equipped with the capability to reconstruct efficiently the neutrino interaction point.

The signature to interpret the result of the test is twofold, i.e. the depletion of the expected count rate (the so called disappearance test) and the observation of the oscillatory spatial pattern (waves) in the profile of the detected events which would be induced by the active-to-sterile oscillation, a true unquestionable smoking gun, if observed.

As unavoidable, there are pros and cons implied by the use of a radioactive source. The advantages are the small size ( one litre), well suited for waves detection, the negligible background from the source itself, obtainable with adequate shielding, the possibility to perform the experiment deep underground, virtually avoiding the $\mu$-induced background, the option to exploit already existing, well known and well characterized detectors (as Borexino), the known, or, measurable neutrino (or antineutrino) spectrum, and the short distance that can be attained from the detector (4 $\mathrm{m}$ in SOX).

On the other hand, the disadvantages are that data can be taken for a limited time, since the useful isotopes have all limited lifetime, the neutrino flux cannot attain that of reactors (in case of SOX the maximum activity is $150 \mathrm{kCi}$ mainly limited by the heat production), and moreover there are difficulties to make the source, to get the authorizations, to perform the transport from the production to the experimental site, and then to dispose it at the end of the experiment. Anyhow, despite these difficulties, the advantages outperform the disadvantages, especially thanks to the unambiguous interpretation of the measurement output, enabled also by the limited background, which can be achieved.

It is worth to remind that the tests mentioned in the previous $\S$ which are at root of the origin of the Gallium anomaly are already very successful examples of deployment of strong radioactive sources within neutrino detectors, in that case solar neutrino detectors. Specifically, Sage and Gallex adopted a ${ }^{51} \mathrm{Cr}$ (both) and a ${ }^{37} \mathrm{Ar}$ source (only Sage) to verify and prove their capability to measure precisely the artificial neutrinos stemming from the deployed source assemblies.

In the current experimental panorama concerning the light sterile neutrinos, while several conceptual proposals have been shaped based on the exploitation of high intensity sources, only SOX has matured and evolved to a concrete project [4], which has reached an advanced phase of realization.

\section{General characteristic of the SOX program}

The core of the SOX (Short Oscillation with BoreXino) program is thus the deployment of an intense anti-neutrino source close to the Borexino detector to test the effect of the putative oscillation $v_{\mathrm{e}} \rightarrow v_{\mathrm{s}}$, which should originate in the liquid scintillator target peculiar and unmistakable spatial waves. 
We shortly describe here, therefore, the two main ingredients of the program, the source and the Borexino detector.

\subsection{The SOX anti-nu source}

SOX will exploit in a first instance a ${ }^{144} \mathrm{Ce}-{ }^{144} \mathrm{Pr}$ anti- $v_{\mathrm{e}}$ source, with the possibility of a further second phase based on ${ }^{51} \mathrm{Cr}$. First proposed in [5], such a source can be made by extracting $\mathrm{Ce}$ from exhausted nuclear fuel and by pressing the resulting $\mathrm{CeO}_{2}$ powder within a properly designed stainless steel capsule. A few $\%$ of the powder will consist of ${ }^{144} \mathrm{Ce}$, being few grams enough to provide the necessary activity; so, the total amount of $\mathrm{CeO}_{2}$ will be of the order of $1 \mathrm{~kg}$.

The capsule will be then sealed according to international regulations for the use and transportation of radioactive materials and inserted into a very think tungsten container (minimum thickness $19 \mathrm{~cm}$ ) to strongly attenuate all unwanted radiations (mainly $\gamma$ rays). Stringent requirements are established on the purity of the $\mathrm{CeO}_{2}$, to limit also the emission of neutrons. The source will be manufactured by the PA Mayak company in Russia and delivered to Gran Sasso by means of a special container certified for the transportation of high activity radioactive materials. The shipment from the manufacturing site in Russia will happen by train to St. Petersburg, by ship to France, and then by truck from France to Gran Sasso.

The Cerium, characterized by a half life of 285 days, decays to Praseodymium which in turn decays almost immediately (17 minutes) to Neodymium 144, emitting anti-neutrinos up to $3 \mathrm{MeV}$. Thus, the Praseodymium anti-neutrinos with energy beyond the IBD threshold of 1.8 $\mathrm{MeV}$ are detected in the liquid scintillator, originating the signals whose detection and analysis are the tools to perform the SOX sterile investigation.

The source will be precisely calibrated (at $1 \%$ level or better) by measuring with a high accuracy thermal calorimeter the heat that it will release, to maximize the sensitivity of the disappearance test. Instead, in order to improve the oscillatory test, the anti-neutrino energy spectrum will be carefully characterized with dedicated experiments. Both characterization are very important, the former to be able to translate the calorimetric measurement into an uncertainty on the anti-neutrino flux above detection threshold, and the latter to take properly into account the spectral shape of the emitted anti-neutrino in the wave analysis.

\subsection{Borexino}

Borexino [6] is a liquid scintillator detector, which employs as active detection medium almost 300 tons of pseudocumene-based scintillator. The intrinsic high luminosity of the liquid scintillation technology is the key toward the goal of Borexino, the real time observation of sub$\mathrm{MeV}$ solar neutrinos through ve elastic scattering, being the ${ }^{7} \mathrm{Be}$ component the main target at the time of the design. However, the lack of directionality of the method makes it impossible to distinguish neutrino scattered electrons from electrons due to natural radioactivity, thus leading to the other crucial requirement of the Borexino technology, e.g. an extremely low radioactive contamination of the detection medium, to a degree never reached before. 
The active scintillating volume, Fig. 1, is observed by 2212 PMTs located on a $13.7 \mathrm{~m}$ diameter sphere and is shielded from the external radiation by more than 2500 tons of water and by 1000 tons of hydrocarbon equal to the main compound of the scintillator (pseudocumene), to ensure zero buoyancy on the thin Nylon Inner Vessel containing the scintillator itself. Of paramount importance for the success of the experiment are also the many purification and handling systems, which were designed and installed to ensure the proper manipulation of the fluids at the exceptional radiopurity demanded by Borexino.

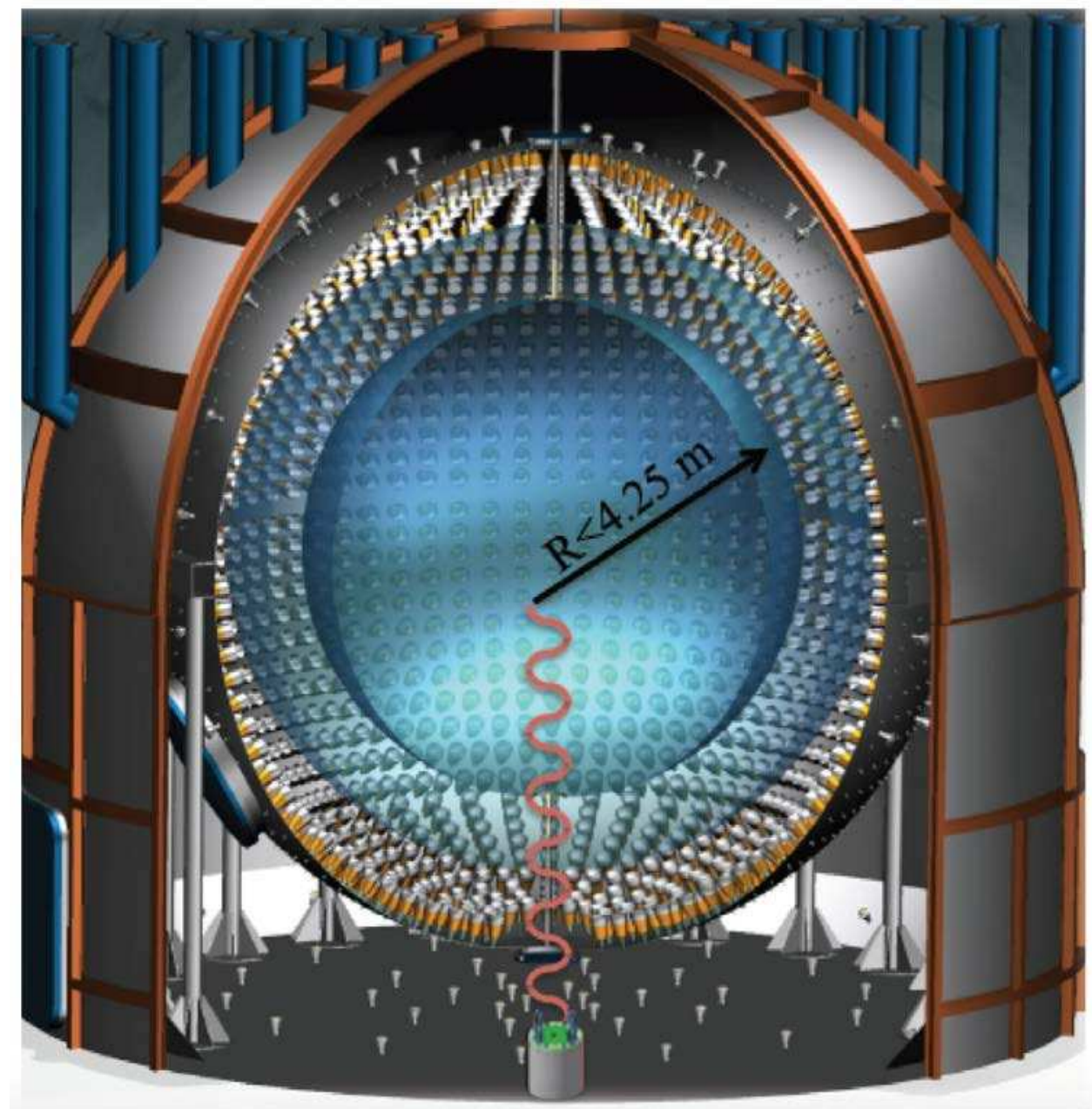

Fig. 1 -Schematic view of the Borexino detector

When data taking started in May 2007, it appeared immediately that the daunting task of the ultralow radioactivity was successfully obtained, representing per se a major technological breakthrough, opening a new era in the field of ultrapure detectors for rare events search. The achieved ultra-low background implies that, once selected by software analysis the design fiducial volume of 100 tons and upon removal of the muon and muon-induced signals, the recorded experimental spectrum is so clean to show spectacularly the striking feature of the ${ }^{7} \mathrm{Be}$ scattering edge, i.e. the unambiguous signature of the occurrence of solar neutrino detection [7]. 
Moreover, Borexino has also detected an unquestionable geoneutrino signal (i.e. antineutrinos from the radioactivity inside the Earth), contributing to pave the way to a complete new method to investigate the interior of our planet [8].

The experiment's characteristics which made possible these accomplishments, essentially the extremely high radiopurity, the stability of the detector and the perfect understanding of its response gained with a detailed MC contrasted with the results from a thorough calibration campaign, are such to make Borexino the perfect playground for an unambiguous source based test aimed to investigate the beyond-standard-model putative sterile neutrino state. This was the consideration that triggered the birth of the SOX program. The source will be conveniently allocated in a tunnel realized during the construction stage of the experiment just beneath the Water Tank.

\section{Sensitivity}

SOX will be at the same time a standard neutrino disappearance experiment and an innovative experiment for the direct detection of oscillation waves.

As already stressed, neutrino oscillations to invisible components (e.g. sterile neutrinos) effectively modulate the distribution of the events within the detection volume in a way that can unambiguously prove their occurrence. Fig. 2, taken from [9], shows the very specific dependence of the event count rate upon the distance from the source and upon the anti-neutrino energy. Such a pattern may prove the existence of oscillations to the sterile partner beyond any reasonable doubt.

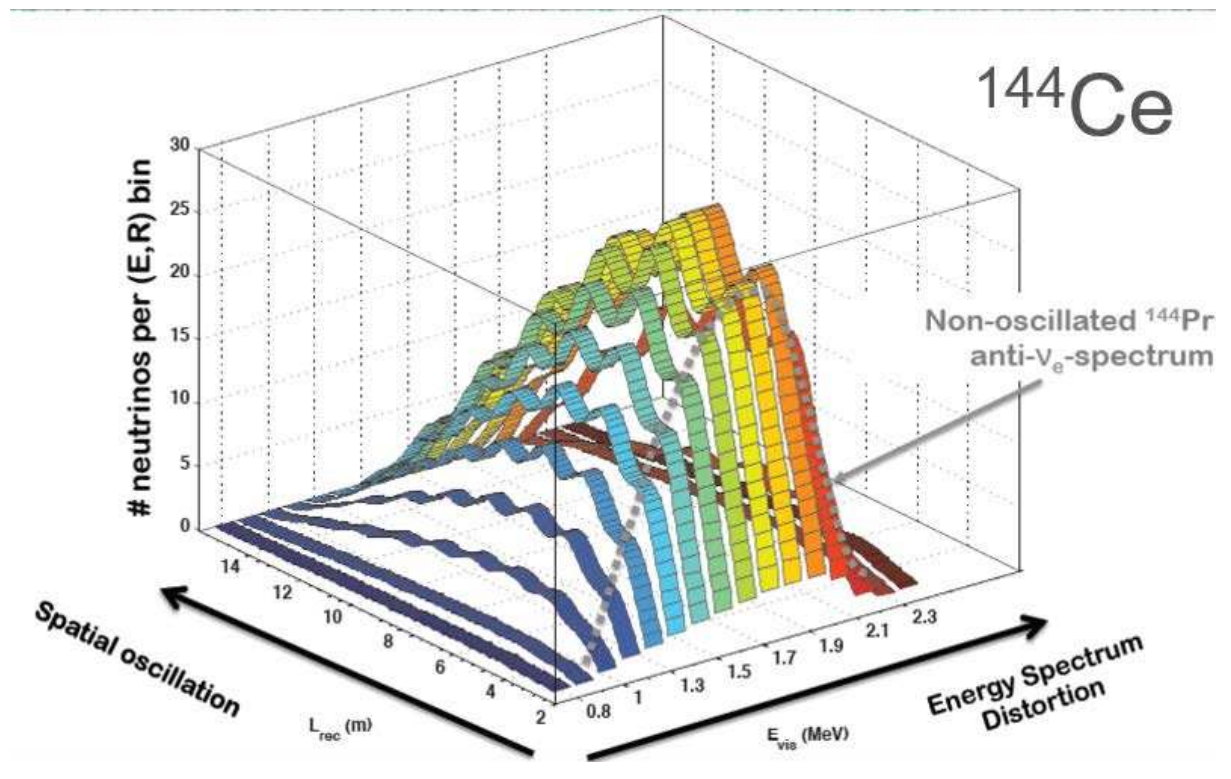

Fig. 2 - The predicted count rate distribution in Borexino as a function of the distance from the anti-neutrino source and of the visible energy

In Fig. 3, the red and blue bands show, respectively, the 95\% C.L. exclusion contours obtained by means of the disappearance test and of the wave analysis. The width of the bands is 
dictated by the minimum $(100 \mathrm{kCi})$ and maximum $(150 \mathrm{kCi})$ activity considered for the source, the real one is expected to lie in between.

The black band is the overall sensitivity plot, obtained combining the red and blue contours. The calculation has been performed assuming the uncertainty of $1.5 \%$ on the source intensity. and a data taking period of 1.5 years. By comparing the black band with the global allowed region stemming from the joint analysis of all the anomalies [10] (grey and light grey contours; a more recent result is reported in [11]) it stems that SOX can test and exclude a very large portion of such a region, included the best fit point. Thus, SOX will be a powerful probe able to shed substantial light to the current light sterile neutrino puzzle. For more details see also [4] and [12].

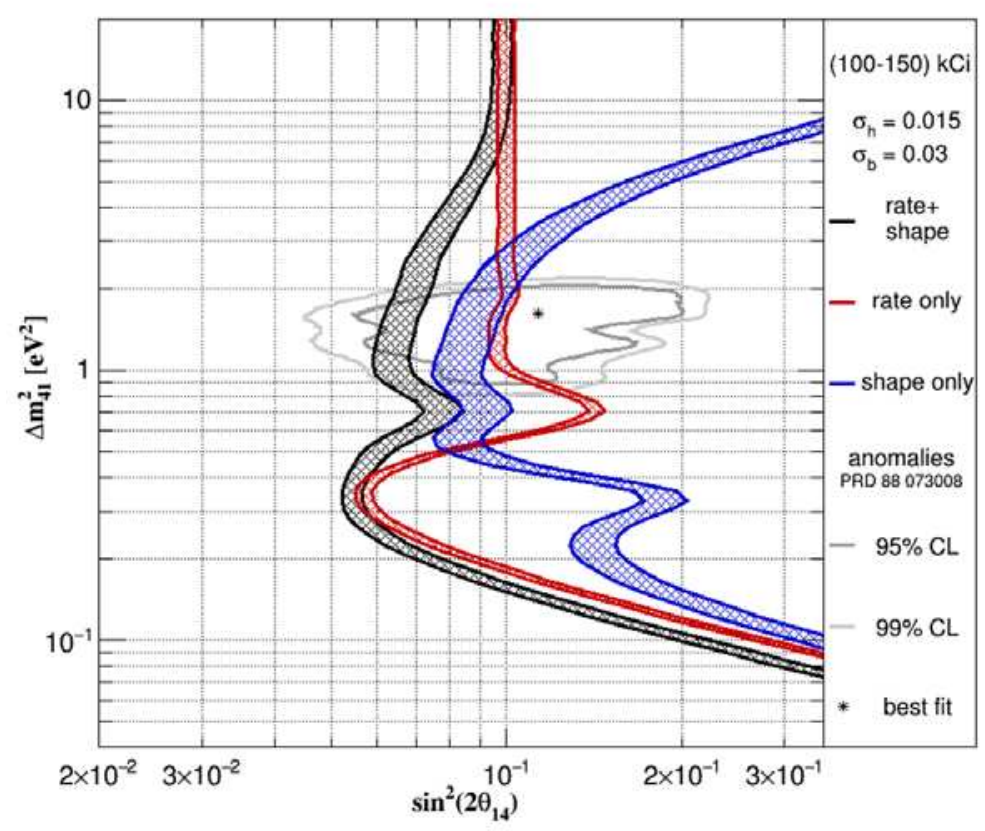

Fig. 3 - The sensitivity of the SOX experiment compared to the allowed region stemming from the global analysis of all the experimental anomalies

\section{Schedule and outlook}

The delivery of the source at Gran Sasso is currently foreseen for January 2018 and data taking will continue for 18 months, so the first physics results will arrive in late 2018 or 2019.

The SOX project includes also a neutrino program by means of a ${ }^{51} \mathrm{Cr}$ source, similar to that used in the 90 s by the Gallex and SAGE collaborations. The enriched ${ }^{50} \mathrm{Cr}$ metal is available in Italy and a feasibility study is in progress to understand whether there is a viable way to irradiate the material and deliver to Gran Sasso a 2-4 MCi source. This part of the project is currently not funded and will be considered after the completion of the Ce based test. 


\section{Acknowledgments}

The author wishes to thank the organizers for the invitation to contribute to such an interesting and enlightening conference.

\section{References}

[1] A. Aguilar et al. (LSND Collaboration), Phys. Rev. D 64 (2001) 112007

[2] G. Mention et al., Phys. Rev. D 83 (2011) 073006

[3] C. Giunti and M. Laveder, Phys. Rev. C 83 (2011) 065504

[4] G. Bellini et al. (Borexino Collaboration), JHEP 08 (2013) 038

[5] M. Cribier et al., Phys. Rev. Lett. 107 (2011) 201801

[6] G. Alimonti et al. (Borexino Collaboration), Nucl. Instr. and Meth. A 600 (2009) 568

[7] G. Bellini et al. (Borexino Collaboration), Phys. Rev. Lett. 108 (2012) 051302

[8] G. Bellini et al. (Borexino Collaboration), Phys. Lett. B 687 (2010) 299

[9] J. Gaffiot et al., Phys. Rev. D 91 (2015) 072005

[10] C. Giunti, M. Laveder, Y. F. Li, and H. W. Long. Phys. Rev. D 88 (2013) 073008

[11] S. Gariazzo et al., J. Physics G 43 (2016) article id. 033001

[12] M. Cribier et al., Phys. Rev. Lett. 107 (2011) 201801 